\title{
The Initial Tangent of the Femoral Arterial Pressure Increase Is an Estimate of Left Ventricular Contractility in Patients Undergoing Cardiacsurgery
}

\author{
Matthias Längin, Christian Kowalski, Frank Christ, Bernhard Zwissler, \\ Hille Kisch-Wedel \\ Department of Anesthesiology, Ludwig Maximilians University, Munich, Germany \\ Email: hkisch@med.uni-muenchen.de
}

Received 9 July 2014; revised 11 August 2014; accepted 3 September 2014

Copyright (C) 2014 by authors and Scientific Research Publishing Inc.

This work is licensed under the Creative Commons Attribution International License (CC BY). http://creativecommons.org/licenses/by/4.0/

(c) (i) Open Access

\begin{abstract}
Purpose: Assessment of contractile function is a major challenge in patients with left ventricular dysfunction, especially during cardiac surgery. The initial tangent of the femoral arterial pressure increase $\left(\tan _{\text {in }}\right)$ has recently been described to be an estimate of left ventricular (LV) contractility. To confirm these findings $\tan _{\text {in }}$ was compared to various indices of $\mathrm{LV}$ performance in patients undergoing cardiac surgery. Methods: Data from 17 patients were evaluated retrospectively. Myocardial performance was estimated by the echocardiographic indices ejection fraction (EF), shortening fraction (FS), circumferential fiber shortening velocity (Vcf), the parameters of pulse contour analysis area under the curve (AUC) and $\tan _{\text {in. }}$. Measurements were taken before and after cardiopulmonary bypass (CPB). Results: Tan $_{\text {in }}$ increased significantly $(813 \pm 216 \mathrm{mmHg} / \mathrm{s} \mathrm{vs.} 1490$ $\pm 450 \mathrm{mmHg} / \mathrm{s}, \mathrm{p}<0.05)$ after CPB, as well as Vcf $(0.89 \pm 0.14 \mathrm{circ} / \mathrm{s}$ vs. $1.47 \pm 0.27 \mathrm{circ} / \mathrm{s}, \mathrm{p}<0.05)$ and EF $(65 \% \pm 7 \%$ vs. $74 \% \pm 6 \%$, p < 0.05$)$. FS did not change $(40.7 \% \pm 7 \%$ vs. $46.5 \% \pm 5 \%$, $p=$ 0.30). AUC significantly dropped after CPB (435 $\pm 54 \mathrm{mmHg}^{*} \mathrm{~s}$ vs. $\left.263 \pm 27 \mathrm{mmHg}^{*} \mathrm{~s}\right)$. Tan $\mathrm{T}_{\text {in }}$ and Vcf correlated strongly $(r=0.70, p<0.001)$, while $\tan _{\text {in }}$ showed only weak correlation with $E F(r=$ $0.36, p=0.037)$. There was no significant correlation with $F S(r=0.31, p=0.079)$. $\operatorname{Tan}_{\text {in }}$ and AUC correlated inversely $(r=-0.62, p<0.001)$. Conclusions: While showing little or no correlation with EF and FS respectively, $\tan _{\text {in }}$ correlated well with the less preload-dependent parameter Vcf, thus suggesting that $\tan _{\text {in }}$ may be used as an easily accessible estimate of LV contractility during cardiac surgery.
\end{abstract}

\section{Keywords}

Arterial Pressure Wave Form Analysis, Left Ventricular Contractility, Cardiacsurgery

How to cite this paper: Längin, M., Kowalski, C., Christ, F., Zwissler, B. and Kisch-Wedel, H. (2014) The Initial Tangent of the Femoral Arterial Pressure Increase Is an Estimate of Left Ventricular Contractility in Patients Undergoing Cardiacsurgery. Open Journal of Anesthesiology, 4, 207-216. http://dx.doi.org/10.4236/ojanes.2014.49030 


\section{Introduction}

Intraoperative assessment of LV contractility in cardiac surgery is of great importance to evaluate the patient's hemodynamic condition and to guide appropriate treatment. Impairment of myocardial function may occur at various stages during cardiac surgery, especially during separation from cardiopulmonary bypass (CPB) [1].

The most widely used index of LV contractility is the maximal first derivate of LV pressure with respect to

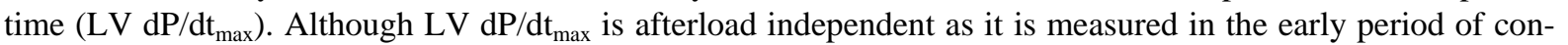
traction, it is considerably dependent on preload changes [2]. In addition, assessment of $\mathrm{LV} \mathrm{dP/dt} \mathrm{dax}_{\max }$ requires an invasive left cardiac catheter, limiting its use outside the catheter laboratory [3].

Transesophageal echocardiography (TEE) has become a standard monitoring tool in cardiac surgery [4]. Ejection phase indices such as systolic shortening fraction (FS) and ejection fraction (EF) are most commonly used in clinical practice to estimate myocardial performance, but are strongly load-dependent [2] [3]-[5]. Circumferential fiber shortening velocity (Vcf), a variable relatively independent to preload changes, has been described years ago, but is rarely used in clinical practice [2] [6]. Calculations of cardiac indices using TEE are time-consuming, even if done by an experienced investigator and thus can only be described as semi-continuous. Furthermore, due to visual interpretation of cardiac function, TEE is prone to subjectivity and poor reproducibility, especially when cardiac function is only roughly estimated via "eyeballing" [7].

In recent years, pulse wave analysis has gained importance in clinical routine. In pulse contour models, individual stroke volume (SV) and cardiac output (CO) are derived from AUC of the arterial pressure curve during LV ejection phase [8]. Measurements are taken continuously and minimally invasively via an arterial catheter, and changes in hemodynamics may thus be observed and instantly reacted to. In contrast to echocardiography, pulse wave analysis is observer-independent. However, as SV and CO are strongly influenced by cardiac loading conditions, they do not represent suitable estimates of cardiac contractility [4]. The rate of pressure increase of the arterial pressure curve, expressed as either the initial tangent on the slope $\left(\tan _{\text {in }}\right)$ or the maximum first derivative $\left(\mathrm{dP} / \mathrm{dt}_{\max }\right)$, are potentially helpful, but scientifically not very well evaluated parameters of LV performance, which can be easily calculated by pulse contour analysis. In previous studies, the pressure increase of the arterial curve correlated well with various indices of LV contractility [9]-[11], however, so far neither $\tan _{\text {in }}$ nor $\mathrm{dP} / \mathrm{dt}_{\max }$ have been evaluated in patients undergoing cardiac surgery. The purpose of the present study was to investigate, whether $\tan _{\text {in }}$ could be used as an estimate of left ventricular contractility during cardiac surgery. To test this hypothesis, the initial tangent of the femoral arterial pressure increase $\left(\tan _{\text {in }}\right)$ was compared with various echocardiographic LV contractility parameters (EF, FS, Vcf) before and after cardiopulmonary bypass.

\section{Materials and Methods}

\subsection{Study Population}

The study was performed retrospectively in accordance with the regulations of the Ethical Committee of the Ludwig Maximilian University, Munich, Germany. 17 patients having received elective cardiac surgery during February and March 2011 at the Ludwig Maximilian University, Campus Grosshadern, were included into the study. Inclusion criteria were scheduled cardiac surgery with cardiopulmonary bypass, the availability of a systematic TEE evaluation, and invasive femoral-arterial pressure measurement. Patients with poor quality echocardiographic images, atrial fibrillation or contraindications for either TEE or femoral arterial catheterization were excluded.

\subsection{Anesthesia}

Anesthesia and catheterization were performed according to internal standards of the Cardiothoracic Anesthesia Division of the Department of Anesthesiology. Routine monitoring included a 5-lead electrocardiogram, pulse oximetry, capnography, invasive measurement of arterial blood pressure by a femoral arterial catheter, central venous pressure, and TEE. Anesthesia was induced with midazolam (5 - $10 \mathrm{mg})$, etomidate (10 - $20 \mathrm{mg})$, sufentanil $(50-100 \mu \mathrm{g})$ and rocuronium $(50 \mathrm{mg})$, and maintained with isoflurane (1.0 - $2.0 \mathrm{vol} \%$ end-expiratory) and sufentanil (50 - $100 \mu \mathrm{g} / \mathrm{h})$. Patients received the catecholamines norepinephrine, epinephrine and/or the phospodiesterase III inhibitor milrinone in order to maintain cardiovascular stability. After CPB, an atrial and/or ventricular pacing was performed if bradycardia or atrioventricular block occurred. 


\subsection{Measurements and Calculations}

Transoesophageal cardiac echocardiographic (TEE) examinations were performed by the cardiac anesthesiologist using a Vivid i system (GE Healthcare, Munich, Germany). Measurements of left ventricular performance were taken throughout the surgery and recorded on an internal hard drive as dicom files. The arterial blood pressure curve was measured by a fluid-filled femoral-arterial catheter system and recorded digitally on a computer via an 16 bit analog/digital converter (sampling rate $250 \mathrm{kHz}$ ). Measuring time points analyzed for this study were hemodynamically stable states directly before opening and after closure of the thoracic cave. Intraoperatively recorded loops of standard transgastric short-axis view as well as midesophageal four-chamber and long axis views were processed to calculate systolic shortening fraction (FS) and biplane ejection fraction (EF). M-mode pictures of the left ventricle were examined to calculate the fractional shortening velocity (Vcf). Postoperative analysis of echocardiographic recordings was performed using the Vivid i system and MicroDicom analysis software (MicroDicom, Sofia, Bulgaria).

Calculation of fractional shortening (FS): Maximal end-diastolic (EDD) and minimal end-systolic (ESD) diameters were obtained through analysis of M-mode loops of standard transgastric short axis views generated and stored before and after CPB (GE Vivid i software). FS was calculated using Equation (1):

$$
\mathrm{FS}=(\mathrm{EDD}-\mathrm{ESD}) / \mathrm{EDD}[\%]
$$

Calculation of ejection fraction (EF): Echocardiographic loops of standard midesophageal four-chamber and long axis views recorded before and after CPB were processed via the GE Vivid i system to calculate systolic biplane ejection fraction (EF) from enddiastolic (EDV) and endsystolic volume (ESV) using the modified Simpson's rule as described elsewhere [12] [13], following in principle Equation (2):

$$
\mathrm{EF}=(\mathrm{EDV}-\mathrm{ESV}) / \mathrm{EDV}[\%]
$$

Calculation of Vcf: M-mode loops of standard transgastric short axis views generated and stored before and after CPB were semi-automatically evaluated with image analysis software (MicroDicom, Sofia, Bulgaria). Maximal end-diastolic (EDD), minimal end-systolic (ESD) diameters, and ejection time (ET), defined as the time from minimal to maximal isotonic contraction of the left ventricle, were obtained to calculate Vcf as depicted in Figure 1 and Equation (3):

$$
\mathrm{Vcf}=(\mathrm{EDD}-\mathrm{ESD}) /(\mathrm{EDD} * \mathrm{ET})[\mathrm{circ} / \mathrm{s}]
$$

Calculation of $\tan _{\text {in }}$ : The recorded arterial pressure curve was processed postoperatively by measurement software (DASYlab 11.0, Measurement Computing, Norton, USA). The initial tangent $\tan _{\text {in }}$-defined as the tangent of the initial pressure increase until the first inclination change [9] as depicted in Figure 2-was fitted

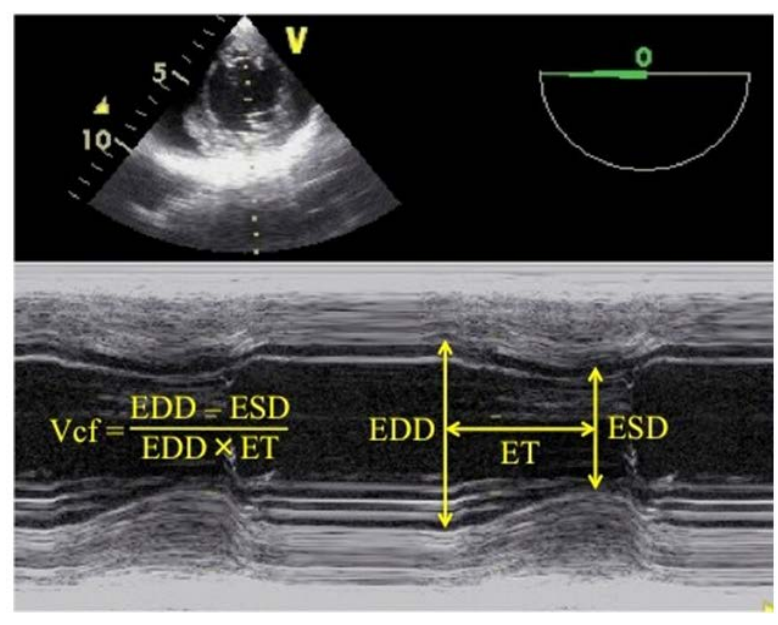

Figure 1. Calculation of circumferential fiber shortening velocity by MMODE transesophageal echocardiography. 


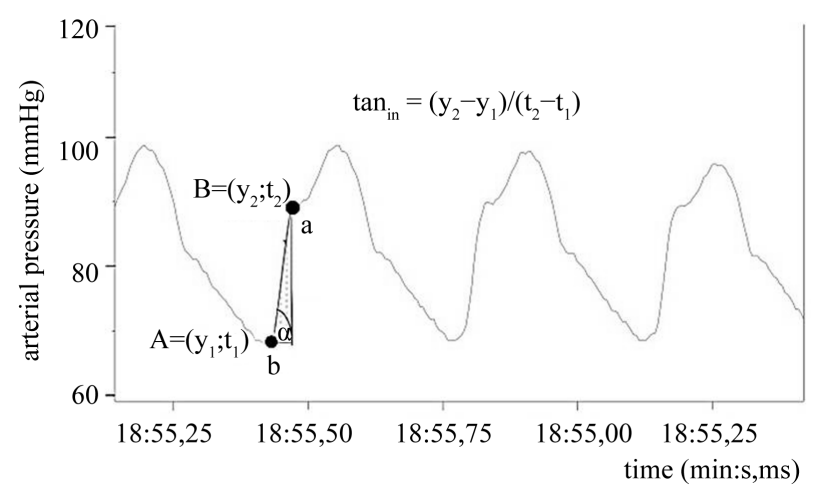

Figure 2. Calculation of the initial tangent of the femoral arterial pressure increase $\left(\tan _{\text {in }}\right)$.

manually to the arterial pressure curve by an investigator blinded to the echocardiographic examinations, and then calculated semi-automatically using Equation (4).

$$
\tan _{\text {in }}=\tan \alpha=\mathrm{a} / \mathrm{b}=\left(\mathrm{y}_{2}-\mathrm{y}_{1}\right) /\left(\mathrm{t}_{2}-\mathrm{t}_{1}\right)[\mathrm{mmHg} / \mathrm{s}]
$$

Calculation of AUC: The area under the curve (AUC), defined as the integral of the arterial pressure, was fitted manually to the initial pressure increase of the femoral arterial pressure curve until the dicrotic notch (investigator blinded to the echocardiographic measurements), and then calculated semi-automatically (DASYlab 11.0, Measurement Computing, Norton, USA).

Measurement of each variable was repeated to improve accuracy. For echocardiographic imaging (FS, EF, Vcf), the mean value of three measurements was calculated. For pulse contour analysis parameters $\left(\tan _{\text {in }} \operatorname{and}\right.$ AUC), the results of ten subsequent pressure waves were averaged.

\subsection{Statistical Analysis}

Data were presented as median \pm semi-interquartile range, as not all of the data were normally distributed (Kolmogorov-Smirnov-test). Correlation was tested by the Spearman rank correlation, and a significance level below 0.05 was considered to be statistically different. Differences between measurements before and after CPB were investigated by the median test and $\mathrm{p}<0.05$ was considered to be statistically different.

\section{Results}

Data from 17 patients (Table 1) undergoing cardiac surgery with cardiopulmonary bypass were analyzed. Surgery included coronary artery bypass graft (CABG) surgery, aortic and mitral valve replacement or reconstruction, and replacement of the ascending aorta (Table 2). Echocardiographic and pulse contour analysis parameters, basic measurements and vasopressor/inotropic support before and after cardiopulmonary bypass (CPB) are shown in Table 3. FS did not change $(40.7 \% \pm 7 \%$ vs. $46.5 \% \pm 5 \%$, p $=0.30)$, whereas EF $(65 \% \pm 7 \%$ vs. $74 \%$ $\pm 6 \%, \mathrm{p}<0.05)$ and Vcf $(0.89 \pm 0.14$ circ/s vs. $1.47 \pm 0.27$ circ/s, $\mathrm{p}<0.05)$ increased significantly after CPB. $\mathrm{Tan}_{\text {in }}$ also increased significantly $(813 \pm 216 \mathrm{mmHg} / \mathrm{s}$ vs. $1490 \pm 450 \mathrm{mmHg} / \mathrm{s}, \mathrm{p}<0.05)$. The area under the curve (AUC) did significantly decrease after CPB (435 $\pm 54 \mathrm{mmHg}^{*}$ s vs. $263 \pm 27 \mathrm{mmHg}^{*}$ ) (Figure 3). Tan did not correlate with FS ( $r=0.31, p=0.079)$, but showed weak correlation with EF $(r=0.36, p=0.037)$, and strong correlation with $\operatorname{Vcf}(r=0.70, \mathrm{p}<0.001)$. Additionally, $\tan _{\mathrm{in}}$ correlated inversely with AUC $(\mathrm{r}=-0.62, \mathrm{p}$ $<0.001$ ) (Figure 4, Table 4).

\section{Discussion}

The rate of arterial pressure increase is a rarely investigated parameter of LV performance deduced from pulse wave analysis. Mathematically, the slope of an arterial pressure curve is calculated using the first derivative $\mathrm{dp} / \mathrm{dt}$ of the pressure wave. The derivative of a function can also be described as the slope of a tangent line to the graph, i.e. the arterial curve. Most previous studies used the maximum first derivative ( $\left.\mathrm{dP} / \mathrm{dt}_{\max }\right)$ to describe $\mathrm{LV}$ function [10] [11]. Our group preferred the initial tangent line to the arterial pressure curve $\left(\tan _{\text {in }}\right)$, which is de 
Table 1. Demographic information about the study population, duration of cardiopulmonary bypass (CPB) and crossclamping. Data are displayed as median \pm semi-interquartile range.

\begin{tabular}{ccc}
\hline \multirow{2}{*}{ Patients } & \multicolumn{2}{c}{ Study population } \\
\cline { 2 - 3 } & Number of patients & 17 \\
\hline Male & 11 \\
Female & 6 \\
Age & $65 \pm 7$ years \\
Height & $173 \pm 6 \mathrm{~cm}$ \\
Weight & $76 \pm 13 \mathrm{~kg}$ \\
Time of CPB & $108 \pm 20 \mathrm{~min}$ \\
Time of crossclamping & $73 \pm 19 \mathrm{~min}$ \\
\hline
\end{tabular}

Table 2. Different types of cardiac surgery with cardiopulmonary bypass (CPB) included into the studygraphic information about the study population, duration of cardio-pulmonary bypass (CPB) and crossclamping. Data are displayed as median \pm semi-interquartile range.

\begin{tabular}{cc}
\hline \multirow{2}{*}{ Types of cardiac surgery } & \\
\hline Number of patients & $\mathrm{n}$ \\
\hline Aortocoronary bypass & 10 \\
\cline { 2 - 2 } Mitral valve recontruction/replacement & 3 \\
Aortic valve replacement & 2 \\
Aortoplasty & 1 \\
Aortoplasty and aortic valve replacement & 1
\end{tabular}

Table 3. Results from transesophageal echocardiography, pulse contour analysis, basic measurements and vasopressor/inotropic therapy. For shortening fraction, ejection fraction and circumferential fiber shortening velocity, the mean value of three measurements was calculated. Pulse contour analysis parameters (area under the curve and initial tangent) were averaged using the results of ten subsequent pressure waves. Data are displayed as median \pm semi-interquartile range. ${ }^{*} \mathrm{P}<0.05$ median-test.

\begin{tabular}{|c|c|c|c|}
\hline & pre CPB & post CPB & \\
\hline \multicolumn{4}{|l|}{ Transoesophageal echocardiography } \\
\hline Shortening fraction (\%) & $40 \pm 7$ & $46 \pm 5$ & ns \\
\hline Ejection fraction (\%) & $65 \pm 7$ & $74 \pm 6$ & * \\
\hline Circumferential fiber shortening velocity (circ/s) & $0.89 \pm 0.14$ & $1.47 \pm 0.27$ & $*$ \\
\hline Enddiastolic volume (ml) & $80 \pm 14$ & $75 \pm 17$ & $\mathrm{~ns}$ \\
\hline \multicolumn{4}{|l|}{ Pulse contour analysis } \\
\hline Area under the curve (mmHg*s) & $435 \pm 54$ & $263 \pm 27$ & $*$ \\
\hline Initial tangent (mmHg/s) & $813 \pm 216$ & $1490 \pm 450$ & * \\
\hline Pulse pressure variation (\%) & $4.7 \pm 2.1$ & $5.8 \pm 3.1$ & ns \\
\hline \multicolumn{4}{|l|}{ Basic measures } \\
\hline Mean arterial pressure (mmHg) & $86 \pm 7$ & $73 \pm 6$ & ns \\
\hline Central venous pressure (mmHg) & $11 \pm 3$ & $7 \pm 2$ & $*$ \\
\hline Heart rate $\left(\min ^{-1}\right)$ & $49 \pm 9$ & $90 \pm 8$ & $*$ \\
\hline \multicolumn{4}{|l|}{ Vasopressor and inotropic therapy } \\
\hline Norepinephrine (mg/h) & $0.3 \pm 0.1$ & $0.5 \pm 0.1$ & * \\
\hline Epinephrine (mg/h) & $0.0 \pm 0.0$ & $0.1 \pm 0.1$ & $*$ \\
\hline Milrinone (mg/h) & $0.0 \pm 0.0$ & $0.4 \pm 0.2$ & $*$ \\
\hline
\end{tabular}


biplane ejection fraction (EF)

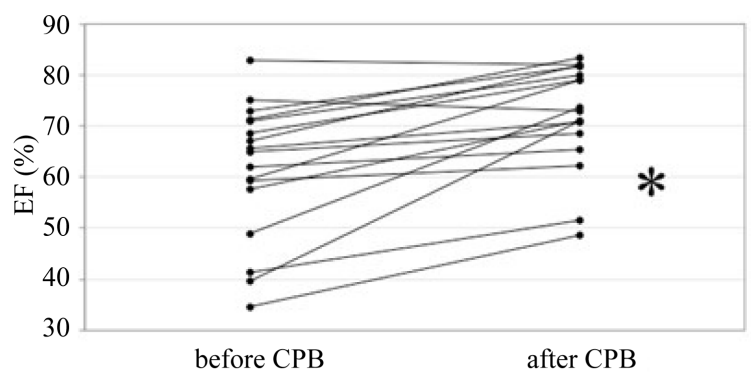

circumferential fiber shortening velocity (Vcf)

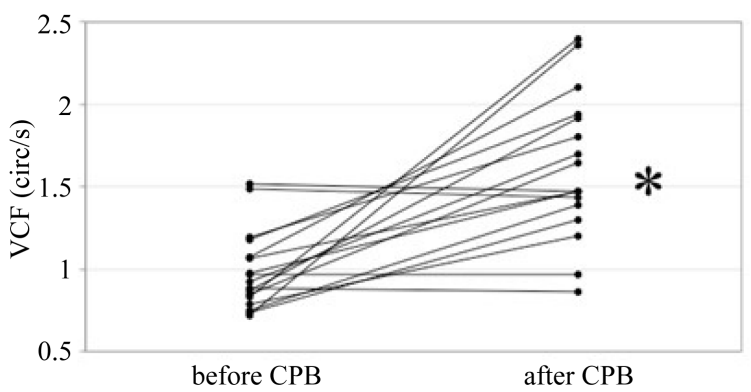

aera under the curve (AUC)

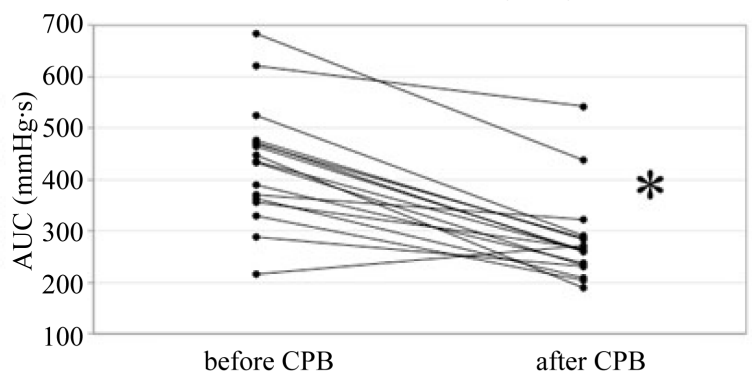

initial tangent $\left(\tan _{\text {in }}\right)$

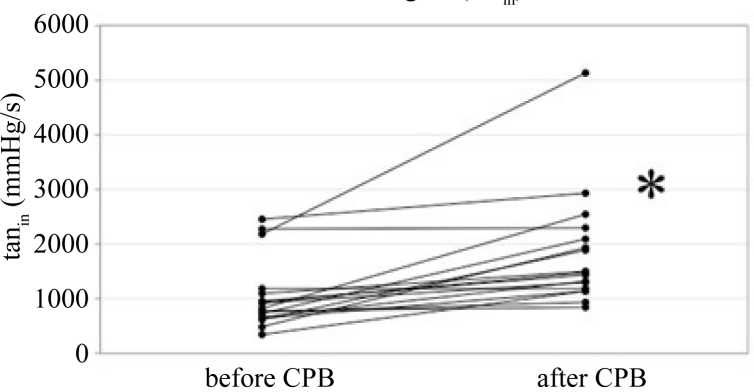

Figure 3. Biplane ejection fraction, area under the curve, circumferential fiber shortening velocity and the initial tangent before and after cardiopulmonary bypass.
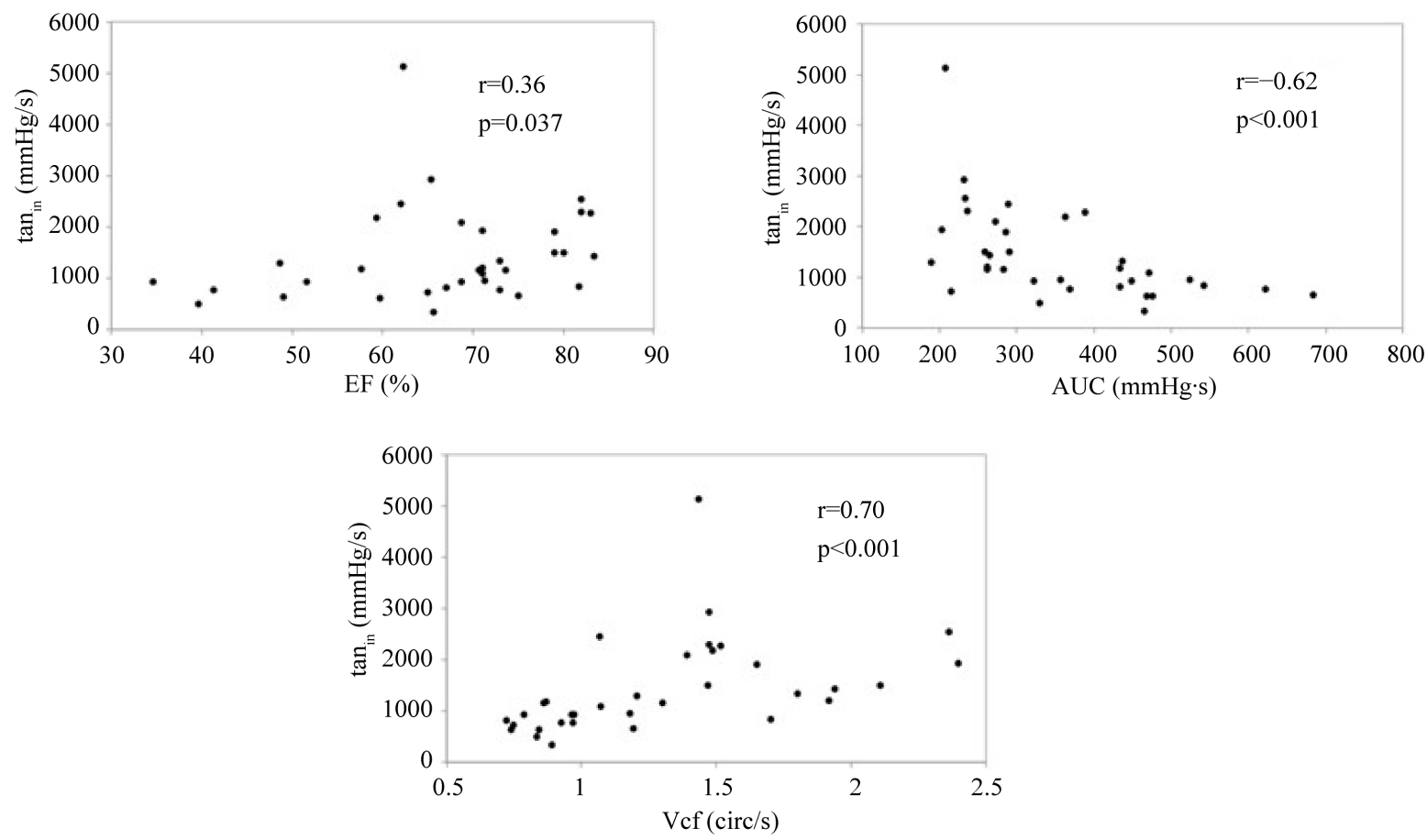

Figure 4. Correlation of the initial tangent $\left(\tan _{\text {in }}\right)$ with left ventricular $(\mathrm{LV})$ ejection fraction $(\mathrm{EF})$, the area under the curve (AUC) and the LV circumferential fiber shortening velocity (Vcf).

fined as the beginning of pressure increase until the first inclination change (Figure 2) [9]. As the rate of pressure increase is supposed to be at its maximum during the early part of the LV ejection phase, $\mathrm{dP} / \mathrm{dt}_{\max }$ and $\tan _{\text {in }}$ 
Table 4. Correlations. Results from transesophageal echocardiography, pulse contour analysis, basic measurements and vasopressor/inotropic therapy. For shortening fraction, ejection fraction and circumferential fiber shortening velocity, the mean value of three measurements was calculated. Pulse contour analysis parameters (area under the curve and initial tangent) were averaged using the results of ten subsequent pressure waves. Data are displayed as median \pm semi-interquartile range. ${ }^{*} \mathrm{P}<0.05$ median-test.

\begin{tabular}{ccccc}
\hline Correlation of & FS $(\%)$ & EF (\%) & Vcf (circ/s) & AUC (mmHg*s) \\
\hline Tan $_{\text {in }}(\mathrm{mmHg} / \mathrm{s})$ with & & & & 0.70 \\
\hline Correlation coefficient & 0.31 & 0.36 & $<0.001$ & $<0.001$ \\
P-value & $\mathrm{ns}$ & 0.037 & $*$ & $*$ \\
\hline
\end{tabular}

should be more or less equivalent. However, there have been no studies directly correlating these two indices yet.

In the present study $\tan _{\text {in }}$ was compared with other parameters of LV performance before and after $\mathrm{CPB}$. EF and $\tan _{\text {in }}$ did significantly increase after CPB, whereas FS did not. In contrast, the area under the curve was significantly smaller after СРВ. Hemodynamic changes after СРВ have been thoroughly investigated in previous research and are not subject of this study [14] [15]. However, changes of $\tan _{\text {in }}$ before and after CPB have never been addressed before. Its significant increase after CPB in accordance with concordant changes of other indices of LV function confirm the assumption that $\tan _{\text {in }}$ might be used as a parameter of LV performance. Interestingly, when comparing $\tan _{\text {in }}$ to the other indices, mainly load-dependent parameters like EF and FS showed either only weak correlation (EF) or none at all (FS). In contrast, the less preload dependent Vcf proved a strong correlation with $\tan _{\text {in. }}$.

To date, little is known about the use of arterial $\mathrm{dP} / \mathrm{dt}_{\max }$ and $\tan _{\text {in, }}$ respectively, for the assessment of LV contractility. De Hert et al. investigated the relationship between $\mathrm{LV} \mathrm{dP} / \mathrm{dt}_{\max }$ and arterial $\mathrm{dP} / \mathrm{dt}_{\max }$ in patients with preserved cardiac function scheduled for CABG surgery [10]. LV dP/dt $\max _{\max }$ tended to be underestimated by arterial $\mathrm{dP} / \mathrm{dt}_{\max }$ in absolute values, but changes in $\mathrm{LV} \mathrm{dP/dt} t_{\max }$ and arterial $\mathrm{dP} / \mathrm{dt}_{\max }$ did correlate very strongly. In-

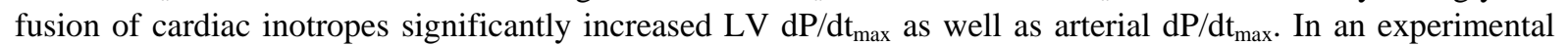
study in pigs, the increase of the aortic pressure curve was compared to endsystolic elastance, the gold-standard for the description of LV contractility [9]. Tan ${ }_{\text {in }}$ significantly correlated with endsystolic elastance, whereas the second part from the dicrotic notch to the pressure peak of the pulse wave did not. Application of beta-agonists led to an increase of $\tan _{\text {in }}$, whereas beta-antagonists lowered $\tan _{\text {in }}$. Morimont et al. could show a significant correlation between endsystolic elastance and arterial $\mathrm{dP} / \mathrm{dt}_{\max }$ in pigs with septic shock [10]. These findings all indicate that $\mathrm{dP} / \mathrm{dt}_{\max }$ and $\tan _{\mathrm{in}}$ can be used as parameters for $\mathrm{LV}$ function.

The extent of load-dependency of arterial $\mathrm{dP} / \mathrm{dt}_{\max }$ and $\tan _{\text {in }}$ remains unclear. De Hert et al. investigated the influence of a preload increase by leg raising on parameters of LV performance. Interestingly, preload change

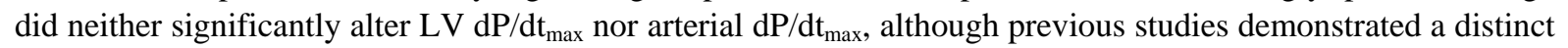
preload dependence of $\mathrm{LV} \mathrm{dP/dt} t_{\max }$ [2] [10]. Morimont et al. detected a relationship between arterial dP/dt $\mathrm{dmax}_{\max }$ and vascular filling: the correlation between endsystolic elastance and arterial $\mathrm{dP} / \mathrm{dt}_{\max }$ increased when vascular filling was adequate, defined as pulse pressure variation (PPV) $<11 \%$ [11]. In this study, preload parameters such as enddiastolic volume and PPV did not change significantly after CPB. Furthermore, mean PPV was well below $11 \%$ before and after $\mathrm{CPB}$, thus implicating that the patients were adequately filled during measurements of $\tan _{\text {in }}$.

$\mathrm{Tan}_{\text {in }}$ as well as arterial $\mathrm{dP} / \mathrm{dt}_{\max }$ are ejection phase indices and must therefore be assumed to be afterload dependent. Arterial pressure increase does most likely not only reflect left ventricular contractility, but is probably also affected by various variables which influence arterial compliance and pulse wave reflection, such as vascular filling conditions, vasoactive drugs, and aortic impedance (Zc). Aging and numerous cardiovascular diseases common in cardiac surgery, e.g. arterial hypertension, lead to aortic stiffening responsible for an increased aortic impedance. Various methods to measure and estimate the individual $Z_{c}$ have been described [8] [16], however, due to the retrospective character of the study, Zc could not be assessed and included into the calculation of $\tan _{\text {in }}$. Arterial compliance has been described to be easily measurable by pulse contour analysis [17]. It seems possible 
to decrease the influence of afterload on $\tan _{\text {in }}$ by including impedance and compliance measurements in future studies.

We did not observe any significant differences of LV function parameters when comparing patients receiving inotropics after CPB to patients who did not (data not shown). Conceivably, patients without the need for inotropic support might already have had good contractility after CPB. However, most patients did receive norepinephrine, a vasopressor with inotropic effects. Furthermore, the difference between both groups might be too small to attain the level of significance due to the size of the study group.

A closer look at the demand for inotropic cathecholamines reveals a peculiar detail: two patients received a repair/replacement of the mitral valve due to high-grade insufficiency. Both of them were treated with dobutamine during the pre-CPB period for hemodynamic stabilization. Initial values of Vcf and $\tan _{\text {in }}$ were considerably larger compared to all other patients. It remains unclear if the reason for this observation lies in elevated contractility or reduced afterload. Interestingly, corresponding differences were not seen in any other indices of LV function.

Certain methodological problems that could not be controlled due to the retrospective character of this study need to be addressed here.

First, invasive blood pressure measurement by fluid filled catheter systems is prone to a multiplicity of errors and artifacts, which can falsify the measurement. The dynamic accuracy of a fluid catheter is affected by resonance and dampening and can lead to either overestimation or underestimation of arterial blood pressure [18]. Imprecise leveling and zeroing as well as contamination of the catheter line such as air bubbles, thrombotic material, and blood may further deteriorate signal quality and accuracy. With worse signal quality, the measurement of $\tan _{\text {in }}$ becomes less accurate. Underdamping might be an explanation for one outlier we observed after cardiopulmonary bypass. In this single case, the slope of the arterial pressure wave was so steep that measurement was almost impossible, thus resulting in extreme values.

Second, Vcf was calculated in M-mode only. Due to the retrospective study design, transgastric short axis loops were not available for a true circumferential measurement of Vcf, so septal and lateral wall motion abnormalities might have been missed by analyses in M-mode. However, with one exception only, none of our subjects had regional wall abnormalities. One patient presented with a septal and anterior hypokinesia, which was limited to the cardiac apex, thus not influencing Vcf measurement in M-mode.

Third, different valve pathologies were included in this study. Aortic stenosis produces a fixed obstruction to left ventricular ejection resulting in a slow initial rise and a late systolic peak in the arterial pressure wave (pulsustardus). By contrast, in aortic regurgitation, the arterial pressure wave rises rapidly. It is likely that especially in aortic stenosis $\tan _{\text {in }}$ underestimates left ventricular contractility by a degree which increases with disease severity. The effect of mitral valve pathologies on the initial tangent remains unclear.

Finally, it is unknown to which extent $\tan _{\text {in }}$ is influenced by heart rhythm. Patients with atrial fibrillation were not included into this study, as clinical experience indicates that $\tan _{\text {in }}$ is not consistent during arrhythmia absoluta. Furthermore, we do not know, if $\tan _{\text {in }}$ is affected by heart rate. Heart rate has been significantly higher after $\mathrm{CPB}$ as patients with bradycardia were paced to ensure sufficient cardiac output. Vcf has also been described to be sensitive to acute alterations in heart rate [19]. All statistic analysis for LV function indices were thus controlled with heart rate corrected parameters using Bazett's formula [20], but the results did not differ significantly from the findings presented here (data not shown). A higher heart rate leading to a shortening of systolic ejection and diastolic filling times, however, might explain why a significant decrease in AUC could be observed after CPB.

\section{Conclusion}

In conclusion, $\tan _{\text {in }}$ correlated well with Vcf, a less preload-dependent measure of left ventricular contractility, whereas $\tan _{\text {in }}$ weakly correlated with biplane EF and did not correlate with FS. These findings suggest that continuous and minimal invasive estimation of LV contractility is easily feasible by measuring $\tan _{\text {in }}$ via a fluid filled femoral arterial catheter system. Moreover, $\tan _{\text {in }}$ might even be superiorly suited for assessing LV contractility as compared to standard observer dependent echocardiographic parameters, such as EF and FS. However, additional prospective studies are required to confirm our results in other clinical settings and to further define the load dependence of $\tan _{\text {in }}$. 


\section{Acknowledgements}

We would like to thank our colleagues from the Division of Cardiothoracic Anesthesiology and Cardiac Surgery, the nursing staff and surgical assistance personnel as well as the intensive care staff for excellent patient care.

\section{Conflict of Interest}

The authors declare that they have no conflict of interest.

\section{References}

[1] Lombard, F.W. and Grichnik, K.P. (2011) Update on Management Strategies for Separation from Cardiopulmonary Bypass. Current Opinion in Anesthesiology, 24, 49-57. http://dx.doi.org/10.1097/ACO.0b013e328342064a

[2] Quinones, M.A., Gaasch, W.H. and Alexander, J.K. (1976) Influence of Acute Changes in Preload, Afterload, Contractile State and Heart Rate on Ejection and Isovolumic Indices of Myocardial Contractility in Man. Circulation, 53, 293-302. http://dx.doi.org/10.1161/01.CIR.53.2.293

[3] Schertel, E.R. (1998) Assessment of Left-Ventricular Function. The Thoracic and Cardiovascular Surgeon, 46, 248254. http://dx.doi.org/10.1055/s-2007-1013081

[4] Fox, J., Glas, K., Swaminathan, M. and Shernan, S. (2005) The Impact of Intraoperative Echocardiography on Clinical Outcomes Following Adult Cardiac Surgery. Seminars in Cardiothoracic and Vascular Anesthesiology, 9, 25-40. http://dx.doi.org/10.1177/108925320500900104

[5] Robotham, J.L., Takata, M., Berman, M. and Harasawa, Y. (1991) Ejection Fraction Revisited. Anesthesiology, 74, 172-183. http://dx.doi.org/10.1097/00000542-199101000-00026

[6] Quinones, M.A., Gaasch, W.H., Cole, J.S. and Alexander, J.K. (1975) Echocardiographic Determination of Left Ventricular Stress-Velocity Relations. Circulation, 51, 689-700. http://dx.doi.org/10.1161/01.CIR.51.4.689

[7] Shiga, T., Wajima, Z., Inoue, T. and Ogawa, R. (2003) Survey of Observer Variation in Transoesophageal Echocardiography: Comparison of Anesthesiology and Cardiology Literature. Journal of Cardiothoracic and Vascular Anesthesia, 17, 430-442. http://dx.doi.org/10.1016/S1053-0770(03)00146-0

[8] Jansen, J.R., Wesseling, K.H., Settels, J.J. and Schreuder, J.J. (1990) Continuous Cardiac Output Monitoring by Pulse Contour during Cardiac Surgery. European Heart Journal, 11, 26-32. http://dx.doi.org/10.1093/eurheartj/11.suppl_I.26

[9] Kisch-Wedel, H., Kemming, G., Meisner, F., Flondor, M., Bruhn, S., Koehler, C. and Zwissler, B. (2008) The Initial Tangent of the Aortic Pressure Increase Is an Estimate of Left Ventricular Contractility in Pigs. Journal of Clinical Monitoring and Computing, 22, 375-380. http://dx.doi.org/10.1007/s10877-008-9145-9

[10] De Hert, S.G., Robert, D., Cromheecke, S., Michard, F., Nijs, J. and Rodrigus, I.E. (2006) Evaluation of Left Ventri-

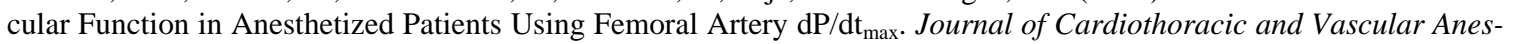
thesiology, 20, 325-330. http://dx.doi.org/10.1053/j.jvca.2005.11.006

[11] Morimont, P., Lambermon, B., Desaive, T., Janssen, N., Chase, G. and D’Orio, V. (2012) Arterial dP/dt $\max$ Accurately Reflects Left Ventricular Contractility during Shock When Adequate Vascular Filling Is Achieved. BMC Cardiovascular Disorders, 12, 13. http://dx.doi.org/10.1186/1471-2261-12-13

[12] McDonald, I.G. (1976) Echocardiographic Assessment of Left Ventricular Function in Aortic Valve Disease. Circulation, 53, 860-864. http://dx.doi.org/10.1161/01.CIR.53.5.860

[13] Otterstad, J.E., Froeland, G., Sutton, M. and Holme, I. (1997) Accuracy and Reproducibility of Biplane Two-Dimensional Echocardiographic Measurements of Left Ventricular Dimensions and Function. European Heart Journal, 18, 507-513. http://dx.doi.org/10.1093/oxfordjournals.eurheartj.a015273

[14] Rhodes, J., Marx, G.R., Tardif, J.C., Romero, B.A., Robinson, A., Acar, P., Pandian, N.G. and Fulton, D.R. (1997) Evaluation of Ventricular dP/dt before and after Open Heart Surgery Using Transesophageal Echocardiography. Echocardiography, 14, 15-22. http://dx.doi.org/10.1111/j.1540-8175.1997.tb00685.X

[15] Gorcsan, J., Gasior, T.A., Mandarino, W.A., Deneault, L.G., Hattler, B.G. and Pinsky, M.R. (1994) Assessment of the Immediate Effects of Cardiopulmonary Bypass on Left Ventricular Performance by On-Line Pressure-Area Relations. Circulation, 89, 180-190.

[16] Chemla, D., Plamann, K. and Nitenberg, A. (2008) Towards New Indices of Arterial Stiffness Using Systolic Pulse Contour Analysis: A Theoretical Point of View. Journal of Cardiovascular Pharmacology, 51, 111-117. http://dx.doi.org/10.1097/FJC.0b013e318163a977

[17] Cohn, J.N. (2001) Arterial Compliance to Stratify Cardiovascular Risk: More Precision in Therapeutic Decision Making. American Journal of Hypertension, 14, 258S-263S. http://dx.doi.org/10.1016/S0895-7061(01)02154-9 
[18] Gardner, R.M. (1981) Direct Blood Pressure Measurement—Dynamic Response Requirements. Anesthesiology, 54, 227-236. http://dx.doi.org/10.1097/00000542-198103000-00010

[19] Hirshleifer, J., Crawford, M., O’Rourke, R.A. and Karliner, J.S. (1975) Influence of Acute Alterations in Heart Rate and Systemic Arterial Pressure on Echocardiographic Measures of Left Ventricular Performance in Normal Human Subjects. Circulation, 52, 835-841. http://dx.doi.org/10.1161/01.CIR.52.5.835

[20] Bazett, H.C. (1920) An Analysis of the Time-Relations of Electrocardiograms. Heart, 7, 353-370. 
Scientific Research Publishing (SCIRP) is one of the largest Open Access journal publishers. It is currently publishing more than 200 open access, online, peer-reviewed journals covering a wide range of academic disciplines. SCIRP serves the worldwide academic communities and contributes to the progress and application of science with its publication.

Other selected journals from SCIRP are listed as below. Submit your manuscript to us via either submit@scirp.org or Online Submission Portal.
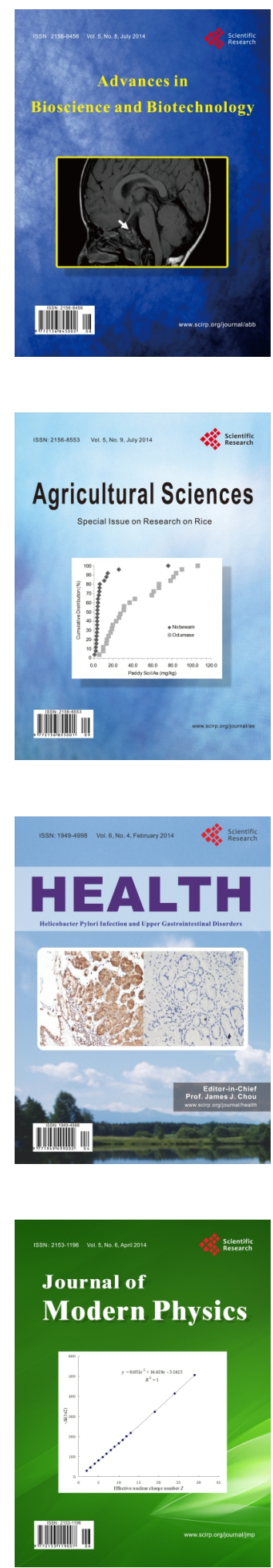
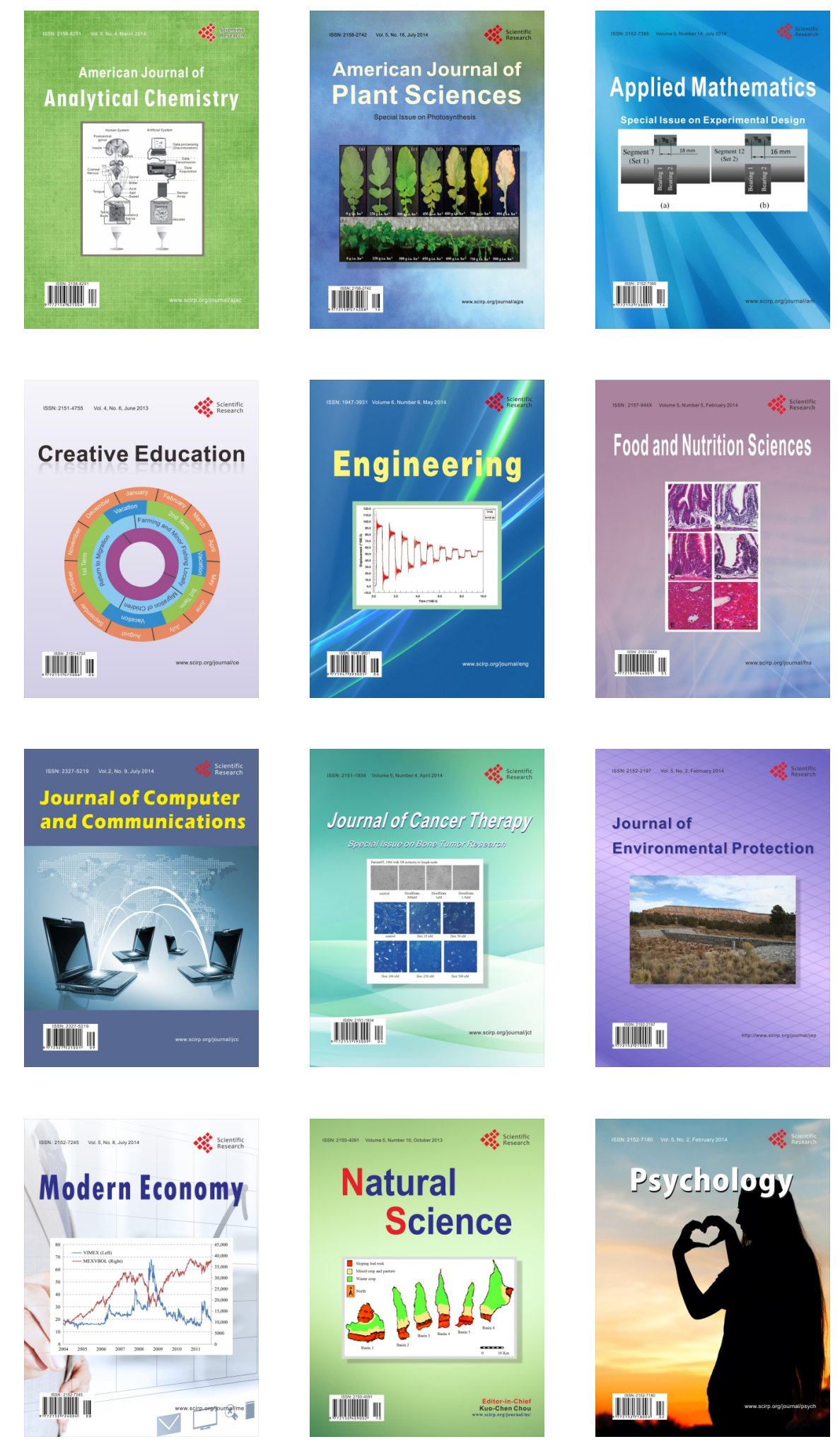